\title{
Duration of colonization with and risk factors for prolonged carriage of multidrug resistant organisms among residents in long-term care facilities
}

I-Wen Lin ${ }^{1}$, Chiao-Yu Huang ${ }^{2,3}$, Sung-Ching Pan ${ }^{4}$, Yng-Chyi Chen ${ }^{5}$ and Chia-Ming Li6 $6^{*}$

\begin{abstract}
Background: Residents of long-term care facilities (LTCFs) colonized with multidrug resistant organisms (MDROs) are often placed under contact isolation to ensure appropriate infection control. This isolation may reduce opportunities for rehabilitation and social stimuli and may also increase medical costs. However, the number of previous studies investigating the duration of colonization in LTCFs is limited. This study was conducted to determine the duration of colonization and risk factors for prolonged carriage of MDROs among residents of a LTCF.
\end{abstract}

Methods: This retrospective study was conducted in a hospital-affiliated nursing home with 59 beds. Fifty-four residents in the nursing home were isolated for MDROs between January 1, 2013 and December 31, 2015. Clinical data were collected from the charts of these 54 residents, including catheter use, colonizing MDRO species and site, underlying diseases, Charlson Comorbidity Index (CCI) scores, and Barthel index scores before and after isolation. Forty-seven residents were included into the statistic analysis. Multivariate Cox regression analyses were performed using duration of colonization as the dependent variable.

Results: The most frequently isolated MDROs were vancomycin-resistant enterococci (VRE) (44.7\%), and the median duration of colonization was 72 (4-407) days. An increased CCl score significantly increased the risk of prolonged colonization (HR: 0.86 , 95\% Cl: 0.76-0.98, $p$ value 0.02).

Conclusion: Among residents of the LTCF, the average duration of MDRO colonization was approximately 3 months. $\mathrm{CCl}$ scores were positively associated with the duration of the MDRO colonization of the LTCF residents. Further studies should be conducted to determine whether implementing isolation protocols for MDRO-colonized LTCF residents is associated with a decline in ADL.

Trial registration: The study protocol was approved by the National Center of Excellence for Clinical Trial and Research of the National Taiwan University Hospital (201512080RINB). The date of registration was December 24, 2015.

Keywords: Colonization, Long-term care facilities, Multidrug-resistant organisms

\footnotetext{
*Correspondence: jeremyli2005@gmail.com

${ }^{6}$ Department of Family Medicine, National Taiwan University Hospital, Bei-Hu

Branch, No.87, Neijiang Street, Taipei City 10845, Taiwan

Full list of author information is available at the end of the article
} 


\section{Background}

The number of residents living in long-term care facilities (LTCFs) has increased due to changes in societal age structures. Residents of LTCFs may be at increased risk of infection as a result of sharing common living areas and participating in group activities. Additionally, due to the frequent hospitalization of LTCF residents, pathogens may be transmitted between LTCFs and hospitals [1-6]. Serious infections may increase medical costs and mortality rates. Residents colonized with multidrug resistant organisms (MDROs) are often placed under contact isolation and prohibited from group activities to ensure infection control. When LTCF staff come into direct contact with a colonized patient or potentially contaminated areas in the resident's environment, frequently employed contact precautions include wearing a gown and using gloves [1, 7-9]. However, isolation may reduce the opportunity for rehabilitation and social stimuli, potentially resulting in functional decline [1]. Thus, LTCFs perform repeated cultures for MDRO pathogens to determine whether isolation may be discontinued.

Risk factors previously identified as potentially associated with an increased probability of microorganism colonization include age, immobility, incontinence, nutrition, functional status, underlying chronic diseases, medication usage (e.g., drugs that affect consciousness, immune functions, gastric acid secretions, and normal flora, including antimicrobial therapy), invasive catheter use, frequency of hospitalization [10-13] and Charlson Comorbidity Index (CCI) score [1].

Several previous studies have investigated the factors that may be associated with the duration of MDRO colonization. For instance, persistent carriage of Klebsiella pneumoniae carbapenemase (KPC)-producing Klebsiella pneumoniae has been found to be associated with catheter use and a low functional status [14], and the risk factors for prolonged carriage of vancomycinresistant enterococci (VRE) that have been previously identified include surgery, antibiotic use at admission, dialysis, and discharge to a nursing home or other health care institution [15]. One study found persistent methicillinresistant Staphylococcus aureus (MRSA) carriage to be associated with admission at another health care institution and disruption of the skin barrier [16]. However, prior studies have predominantly focused on patients admitted to hospitals.

Several previous studies have investigated risk factors for MDRO colonization in LTCFs. A cross-sectional study of 84 nursing home residents in an LTCF in Boston, Massachusetts found that having a diagnosis of advanced dementia was a major risk factor for harboring MDRGN (multidrug-resistant gram-negative bacteria) [17]. Another nested case-control study was conducted in four co-located LTCFs in Australia. Of the 115 residents included in this study, 41 carried MDROs. This study reported that wound management, medical device use and pressure ulcers were independent risk factors for MDRO colonization [18].

Previous studies investigating the duration of MDRO colonization have been predominantly conducted in hospitals $[2,3,6,8,14-16]$. No prior studies have investigated the factors that affect the duration of MDRO colonization in the LTCF setting. However, the implementation of MDRO isolation protocols to control infection may lead to the limitation of resident activities, thereby shortening the golden time for functional recovery after hospitalization. The consequences of contact isolation may also include increased medical costs and increased health care worker (HCW) and caregiver workloads. Information regarding the length of time required for MDRO clearance would be of benefit to both HCWs and families. To answer these questions, we conducted a retrospective observational study to determine the duration of MDRO colonization and risk factors associated with colonization duration in LTCF residents.

\section{Methods \\ Setting}

The setting for this study was a hospital-affiliated nursing home with 59 beds. In this facility, infection control protocols are strictly enforced under the supervision of the hospital infection control center. Urine, sputum, and anal swab samples were collected for bacterial culture from every new resident and from all residents who were readmitted after hospitalization on the date of LTCF admission. If the culture results indicate the presence of MDROs, residents were placed under contact isolation. If the resident had been isolated during hospitalization, the LTCF maintained contact isolation after the resident was transferred back to the LTCF and performed monthly follow-up bacterial cultures to determine whether isolation could safely be discontinued.

The residents with MDRO colonization in this facility were arranged in a single-patient room as a priority. If a single-patient room was not available, residents with the same MDROs would be placed together or lived with residents with a low risk of contracting an infection. In multi-patient rooms, at least $1 \mathrm{~m}$ of spatial separation was provided, and curtains would be used. The caregivers were required to ensure contact precautions, such as wearing a gown and gloves for all interactions that may involve contact with the patient or potentially contaminated areas in the patient's environment.

MDROs are defined as Methicillin-resistant Staphylococcus aureus, Vancomycin-resistant Enterococcus spp., Carbapenem-resistant Enterobacteriaceae spp., or other multi-drug resistant pathogens with non-susceptibility to at least one agent in at least 3 antimicrobial classes 
of the following 6 classes: ampicillin/sulbactam, cephalosporins, $\beta$-lactam $/ \beta$-lactamase inhibitor combination, carbapenems, fluoroquinolones, and aminoglycosides [19]. VRE was initially identified by CHROMagar VRE.

The following criteria were used to determine MDRO resolution: (1) absence of infectious symptoms at the site of original infection/colonization; (2) discontinuation of antibiotics; (3) removal of related catheters; (4) one negative nasal swab culture for MRSA and one negative throat swab culture for multidrug-resistant Acinetobacter baumannii (MDRAB); and (5) three negative anal swab cultures for VRE, and one negative anal swab culture for carbapenem-resistant gram-negative bacteria (CRGNB). MDRO resolution needed to meet 1 to 3, plus 4 or 5 , depending on the species of MDRO.

\section{Enrollment}

We identified the residents of a single LTCF localized in Taipei City, Taiwan from whom a MDRO was isolated from Jan 1, 2013 to Dec 31, 2015. We reviewed the charts of identified patients and extracted clinical and demographic data, including age, sex, catheter use (including nasogastric tube, indwelling urinary catheter,

Table 1 Baseline characteristics of study subjects $(N=47)$

\begin{tabular}{|c|c|c|c|}
\hline Variable & N (\%) & $\begin{array}{l}\text { Duration of colonization, } \\
\text { (Mean } \pm \mathrm{SD}) \text {, days }\end{array}$ & $\begin{array}{l}\text { Duration of colonization, } \\
\text { (Median(Range)), days }\end{array}$ \\
\hline Total & $47(100)$ & $94.6 \pm 75.6$ & $72(4-407)$ \\
\hline Subjects who died before decolonization & $8(17.0)$ & & \\
\hline Age, y & $83.9 \pm 7.8$ & & \\
\hline \multicolumn{4}{|l|}{ Gender, N(\%) } \\
\hline Men & $27(57.4)$ & $92.7 \pm 85.9$ & $63(14-107)$ \\
\hline Women & $20(42.6)$ & $97.2 \pm 61.2$ & $75(4-263)$ \\
\hline \multicolumn{4}{|l|}{ Origin of case, N(\%) } \\
\hline Hospital & $37(78.7)$ & $96.0 \pm 62.8$ & $72(32-314)$ \\
\hline Long term care facility & $10(21.3)$ & $89.6 \pm 115.9$ & $59.5(4-407)$ \\
\hline \multicolumn{4}{|l|}{ Type of indwelling devices, N(\%) } \\
\hline Nasogastric tube & $32(68.1)$ & $97.7 \pm 86.5$ & $72(14-107)$ \\
\hline Foley catheter & $22(46.8)$ & $71.1 \pm 44.6$ & $62(4-158)$ \\
\hline Tracheostomy & $8(17.0)$ & $116.8 \pm 121.2$ & $70.5(32-407)$ \\
\hline \multicolumn{4}{|l|}{ Type of MDROs, N(\%) } \\
\hline VRE & $21(44.7)$ & $108.1 \pm 72.7$ & $80(35-314)$ \\
\hline MRSA & $6(12.8)$ & $100.0 \pm 61.5$ & $111(4-158)$ \\
\hline MDRAB & $7(14.9)$ & $111.1 \pm 137.6$ & $48(14-107)$ \\
\hline CRGNB & $13(27.7)$ & $61.4 \pm 19.5$ & $60(31-111)$ \\
\hline Barthel index score before isolation & $6.4 \pm 20.2$ & & \\
\hline Barthel index score after isolation & $5.1 \pm 17.3$ & & \\
\hline Charlson comorbidity index score & $5.8 \pm 2.5$ & & \\
\hline \multicolumn{4}{|l|}{ Underlying comorbidity, N(\%) } \\
\hline Dementia & 43(91.5) & $96.8 \pm 77.5$ & $72(14-107)$ \\
\hline Cerebrovascular disease & $33(70.2)$ & $85.9 \pm 67.7$ & $62(4-314)$ \\
\hline Cardiovascular diseases & 25(53.2) & $103.6 \pm 72.2$ & $74(4-314)$ \\
\hline Chronic lung disease & $21(44.7)$ & $82.0 \pm 80.9$ & $61(14-407)$ \\
\hline Moderate or severe renal disease & $21(44.7)$ & $85.9 \pm 67.7$ & $72(4-407)$ \\
\hline Diabetes mellitus & $21(44.7)$ & $99.8 \pm 62.5$ & $78(4-314)$ \\
\hline Peptic ulcer disease & 18(38.3) & $93.7 \pm 99.3$ & $59(14-407)$ \\
\hline Cancer & $7(14.9)$ & $148.1 \pm 118.4$ & $128(72-407)$ \\
\hline Moderate or severe liver disease & $4(8.5)$ & $88.3 \pm 30.6$ & $77(66-133)$ \\
\hline
\end{tabular}


and tracheostomy tube), colonizing MDRO species, site of colonization (including sputum, urine, anus, blood, or wound colonization), antibiotics used most recently prior to MDRO detection, chronic illnesses, CCI scores, and Barthel index scores before and after isolation.

The Charlson comorbidity index (CCI) was developed to predict the one-year mortality of patients based on comorbidity information. The CCI included 19 items: myocardial infarction, congestive heart failure, peripheral vascular disease, cerebrovascular disease without sequelae, hemiplegia, dementia, chronic pulmonary disease, connective tissue disease, peptic ulcer disease, mild liver disease, moderate or severe liver disease, moderate or severe renal disease, diabetes without end-organ damage, diabetes with end-organ damage, tumor without metastasis, leukemia, lymphoma, metastatic solid tumor, and acquired immunodeficiency syndrome (AIDS). CCI has been used to predict the outcome and risk of death from many comorbid diseases $[20,21]$.
The Barthel index is a widely used instrument to evaluate the functional status of and the dependency of daily life and includes 10 items: feeding, moving from wheelchair to bed and returning, personal care, getting on and off the toilet, bathing oneself, walking on a level surface, ascending and descending stairs, dressing, controlling bowels, and controlling the bladder [22].

The dates of isolation and resolution were defined by the dates on which positive and negative bacterial cultures were obtained, respectively.

\section{Statistical analysis}

The data were summarized as frequencies or percentages for categorical variables and as median and range (inter-quartile range) for continuous variables. Study subjects were censored at the time of death or loss to follow-up. A time-to-event analysis of the weeks between the date of readmission or admission to the LTCF

Table 2 Cox regression analysis for the probability of MDRO decolonization

\begin{tabular}{|c|c|c|c|c|c|c|}
\hline \multirow[t]{2}{*}{ Variable } & \multicolumn{3}{|c|}{ Univariate regression analysis } & \multicolumn{3}{|c|}{ Multivariate regression analysis } \\
\hline & Raw HR & $95 \% \mathrm{Cl}$ & $P$ value & Adjusted HR & $95 \% \mathrm{Cl}$ & $P$ value \\
\hline $\mathrm{Age}^{\mathrm{C}}$ & 1.00 & $0.96-1.04$ & 0.831 & 1.01 & $0.97-1.06$ & 0.532 \\
\hline Gender $^{a, c}$ & $1.36^{\mathrm{a}}$ & $0.71-2.58^{\mathrm{a}}$ & $0.352^{\mathrm{a}}$ & $1.92^{\mathrm{a}}$ & $0.90-4.09^{a}$ & $0.093^{\mathrm{a}}$ \\
\hline Origin of case $e^{b}$ & $1.26^{\mathrm{b}}$ & $0.57-2.74^{b}$ & $0.569^{b}$ & & & \\
\hline \multicolumn{7}{|l|}{ Type of indwelling devices, N(\%) } \\
\hline Nasogastric tube & 0.97 & $0.50-1.91$ & 0.939 & & & \\
\hline Foley catheter $^{c}$ & 1.69 & $0.88-3.22$ & 0.114 & & & \\
\hline Tracheostomy & 0.82 & $0.34-1.96$ & 0.649 & & & \\
\hline \multicolumn{7}{|l|}{ Type of MDROs, N(\%) } \\
\hline VRE & 0.66 & $0.35-1.26$ & 0.209 & & & \\
\hline MRSA & 0.90 & $0.37-2.18$ & 0.818 & & & \\
\hline MDRAB & 0.88 & $0.34-2.27$ & 0.792 & & & \\
\hline $\mathrm{CRGNB}^{\mathrm{C}}$ & 2.46 & $1.13-5.33$ & 0.023 & & & \\
\hline Charlson comorbidity index score ${ }^{c}$ & 0.89 & $0.79-1.01$ & 0.064 & 0.86 & $0.76-0.98$ & 0.02 \\
\hline \multicolumn{7}{|l|}{ Underlying comorbidity } \\
\hline Dementia & 0.62 & $0.22-1.77$ & 0.367 & & & \\
\hline Cerebrovascular disease & 1.35 & $0.68-2.67$ & 0.389 & & & \\
\hline Cardiovascular diseases & 0.77 & $0.41-1.47$ & 0.434 & & & \\
\hline Chronic lung disease & 1.52 & $0.80-2.90$ & 0.203 & & & \\
\hline Moderate or severe renal disease & 0.82 & $0.43-1.59$ & 0.562 & & & \\
\hline Diabetes mellitus & 0.81 & $0.43-1.52$ & 0.504 & & & \\
\hline Peptic ulcer disease ${ }^{c}$ & 1.57 & $0.82-3.00$ & 0.176 & & & \\
\hline Cancer $^{c}$ & 0.51 & $0.20-1.30$ & 0.156 & & & \\
\hline Moderate or severe liver disease & 1.28 & $0.45-3.68$ & 0.644 & & & \\
\hline
\end{tabular}

MRSA Methicillin-resistant Staphylococcus aureus; MDRAB Multidrug-resistant Acinetobacter baumannii; CRGNB Carbapenem-resistant gram-negative bacilli ${ }^{a}$ Comparison between male and female subjects (reference)

${ }^{b}$ Comparison of cases originating from the long-term care facility and cases originating from hospitals (reference)

cage, gender and variables with a $p$ value less than 0.2 in the univariate model were included in the multivariate model 
and the date of the resolution of MDRO colonization was performed using the Kaplan-Meier method. We performed univariate and multivariate Cox proportional hazard regression analyses in which the duration of colonization was used as the dependent variable. Age, sex and variables with a $p$ value less than 0.2 in the univariate model were included in the multivariate model. A backward stepwise regression analysis was performed. The SPSS version 11.0 statistical package (SPSS Inc., Chicago, IL) was used for analysis. A $p$ value $<0.05$ was considered statistically significant.

\section{Results}

In total, MDROs were isolated from 54 patients during the study period. Seven patients were excluded due to missing data; thus, 47 patients were included in the final analysis, and 8 patients died before MDRO clearance. The mean age of the patients was $83.9 \pm 7.8$ years; 27 patients were male, and 20 were female. The mean Barthel index score was $6.4 \pm 20.2$ before isolation and $5.1 \pm 17.3$ after isolation. Of the 47 included patients, 37 (78.7\%) were colonized with MDROs during hospitalization, whereas the remaining patients were colonized with MDROs at the LTCF. Overall, 89.4\% of the patients had at least one indwelling device. The most frequently isolated MDROs were VRE (44.7\%). The mean CCI score was $5.8 \pm 2.5$. The most prevalent underlying disease among the patients was dementia (91.5\%). The mean duration of colonization was $94.6 \pm 75.6$ days, and the median duration of colonization was $72(4-407)$ days. The median durations of colonization for each pathogen were 80 (35-314) days for VRE, 111 (4-158) days for MRSA, 48 (14-107) days for MDRAB, and 60 (31-111) days for CRGNB. Detailed descriptive statistics are shown in Table 1.

Age, sex, Foley catheter use, CCI score, and CRGNB colonization were included in the model. Multivariate analysis revealed that only an increased CCI score was associated with increased risk of prolonged colonization after accounting for age and sex (HR: 0.86, 95\% CI: $0.76-0.98, p$ value 0.02 ). The origin of MDRO colonization, type of indwelling device, and species of MDRO did not affect the duration of colonization (Table 2). The Kaplan-Meier estimate for the duration of MDRO colonization is shown in Fig. 1.

\section{Discussion}

The median colonization days of MDROs were more than 2 months in this study. In addition, 8 patients died before MDRO clearance. The results of this study suggest that the duration of colonization for MDROcolonized LTCR residents may be longer than expected. Sohn KM, et al. found the median duration of VRE colonization to be 5.57 weeks after hospital discharge [15], and Scanvic A, et al. reported an estimated median time of MRSA clearance of 8.5 months [16]. Overall, large variations are observed in different study settings.

Patients with a higher CCI score had a significantly longer duration of colonization. In these residents, MDRO colonization may be prolonged due to the presence of a greater number of comorbidities. This

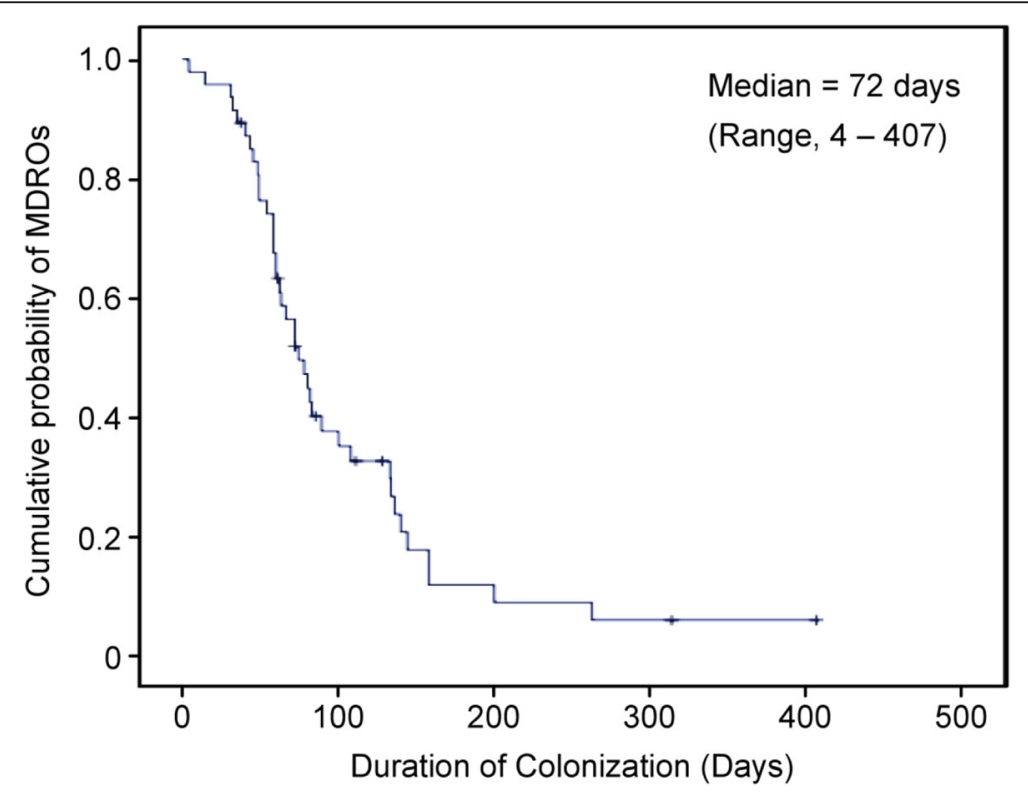

Fig. 1 The Kaplan-Meier estimate for the duration of MDRO colonization 
hypothesis is reasonable because comorbidities may contribute to a more complex disease course or more frail condition, thereby leading to a decrease capacity for MDRO clearance. The risk factors identified in this study were consistent with those of studies conducted in the hospital setting $[2,3,6,8,14-16]$.

We attempted to determine the manner in which the implementation of isolation protocols for MDROcolonized patients affected their activities of daily living (ADL, measured by the Barthel index). However, the ADL scores of the residents included in our study were mostly low, which made a direct evaluation of the correlation between ADL score and isolation duration difficult and limited our ability to assess this issue, as a result. This important issue should be investigated further in multicenter studies that are conducted in LTCFs, that include a large number of patients, and that enroll residents with a wider spectrum of ADL scores.

The risk factors identified in this study are not currently modifiable. However, the findings of this study may influence the isolation protocols implemented for MDRO-colonized patients. As we noticed that MDRO colonization was longer than 2-3 months for those with increased CCI scores, rehabilitation should be maintained even during the isolation period to prevent further functional decline. For example, isolating colonized residents in the same location, sharing the same environment or rehabilitation modality, may retain their rehabilitation program and at the same time prevent further transmission of MDROs to uncolonized residents. Furthermore, identification of the modifiable risk factors that influence the duration of colonization may reduce or even avoid the costs of isolation.

There are some limitations to our study. First, we only included a small number of patients from whom MDROs were isolated. Second, this study was conducted only in a single LTCF, but this single LTCF regularly performed follow-up cultures for MDROs to determine if isolation could be discontinued. Moreover, we simultaneously evaluated multiple types of MDROs, but different MDROs may be associated with different risk factors that affect the duration of patient isolation.

\section{Conclusion}

The average duration of colonization among MDROcolonized LTCF residents was approximately 3 months. Among LTCF residents, CCI scores were positively associated with the duration of colonization. Further studies should be conducted to determine whether implementing the isolation protocols for MDRO-colonized LTCF residents is associated with a decline in ADL.

\section{Abbreviations}

ADL: activities of daily living; CCl: Charlson Comorbidity Index;

CRGNB: carbapenem-resistant gram-negative bacteria; HCW: health care worker; KPC: Klebsiella pneumoniae carbapenemase; LTCFs: long-term care facilities; MDRAB: multidrug-resistant Acinetobacter baumannii; MDRGN: multidrug-resistant gram-negative bacteria; MDROs: multidrug-resistant organisms; MRSA: methicillinresistant Staphylococcus aureus; VRE: Vancomycin-resistant enterococci

\section{Acknowledgements}

The authors would like to thank registered nurse Haiyen Chou and all colleagues of the Nursing home, National Taiwan University Hospital Beihu Branch for data collection.

\section{Funding}

The English editing fee of this paper was supported by a grant from the National Taiwan University Hospital, Beihu Branch, No.10504.

\section{Availability of data and materials}

The data that support the findings of this study are available from National Taiwan University Hospital, but restrictions apply regarding to the availability of these data, which were used under license for the current study and are thus not publicly available. Data are however available from the authors upon reasonable request and with permission of National Taiwan University Hospital.

\section{Authors' contributions}

IWL mainly contributed to chart reviewing and primary article writing. $\mathrm{CYH}$ mainly contributed to statistical analyses. YCC mainly contributed to case enrollment. SCP and CML supervised the study. All authors read and approved the final manuscript.

\section{Ethics approval and consent to participate}

The study protocol was approved by the National Center of Excellence for Clinical Trial and Research of the National Taiwan University Hospital (201512080RINB).

\section{Consent for publication}

Not applicable.

\section{Competing interests}

The authors declare that they have no competing interests.

\section{Publisher's Note}

Springer Nature remains neutral with regard to jurisdictional claims in published maps and institutional affiliations.

\section{Author details}

1Department of Family Medicine, National Taiwan University Hospital, Bei-Hu Branch, No.87, Neijiang Street, Taipei City 10845, Taiwan. ${ }^{2}$ Department of Family Medicine, Renai Branch, No.10, Sec. 4, Ren'ai Rd., Da'an Dist, Taipei City 106, Taiwan, Republic of China. ${ }^{3}$ Institute of Health Policy and Management, National Taiwan University, No. 1, Sec. 4, Roosevelt Rd, Taipei 10617, Taiwan, Republic of China. ${ }^{4}$ Department of Internal Medicine, National Taiwan University Hospital, No.7, Zhongshan S. Rd., Zhongzheng Dist, Taipei City 100, Taiwan, Republic of China. ${ }^{5}$ Department of Nursing, National Taiwan University Hospital, Bei-Hu Branch, No.87, Neijiang Street, Taipei City 10845, Taiwan. ${ }^{6}$ Department of Family Medicine, National Taiwan University Hospital, Bei-Hu Branch, No.87, Neijiang Street, Taipei City 10845, Taiwan.

Received: 23 April 2017 Accepted: 22 August 2017

Published online: 25 August 2017

References

1. Healthcare Infection Control Practices Advisory Committee (HICPAC). Guideline for isolation precautions. http://www.cdc.gov/hicpac/2007IP/ 2007ip_part1.html\#3; 2007. Accessed Jan 25, 2016.

2. Evans RS, Lloyd JF, Abouzelof RH, Taylor CW, Anderson VR, Samore MH. System-wide surveillance for clinical encounters by patients previously identified with MRSA and VRE. Stud Health Technol Inform. 2004;107:212-6.

3. Mylotte JM, Goodnough S, Tayara A. Antibiotic-resistant organisms among long-term care facility residents on admission to an inpatient geriatrics unit: retrospective and prospective surveillance. Am J Infect Control. 2001;29:139-44.

4. Strausbaugh LJ, Jacobson C, Yost T. Methicillin-resistant Staphylococcus Aureus in a nursing home and affiliated hospital: a four-year perspective. Infect Control Hosp Epidemiol. 1993;14:331-6. 
5. Wiener J, Quinn JP, Bradford PA, Goering RV, Nathan C, Bush K, et al. Multiple antibiotic-resistant Klebsiella and Escherichia Coli in nursing homes. JAMA. 1999;281:517-23.

6. Pop-Vicas AE, D'Agata EM. The rising influx of multidrug-resistant gramnegative bacilli into a tertiary care hospital. Clin Infect Dis. 2005;40:1792-8.

7. Donskey CJ. The role of the intestinal tract as a reservoir and source for transmission of nosocomial pathogens. Clin Infect Dis. 2004;39:219-26.

8. Bhalla A, Pultz NJ, Gries DM, Ray AJ, Eckstein EC, Aron DC, et al. Acquisition of nosocomial pathogens on hands after contact with environmental surfaces near hospitalized patients. Infect Control Hosp Epidemiol. 2004;25:164-7.

9. Duckro AN, Blom DW, Lyle EA, Weinstein RA, Hayden MK. Transfer of vancomycin-resistant enterococci via health care worker hands. Arch Intern Med. 2005;165:302-7.

10. High KP, Bradley S, Loeb M, Palmer R, Quagliarello V, Yoshikawa T. A new paradigm for clinical investigation of infectious syndromes in older adults: assessment of functional status as a risk factor and outcome measure. Clin Infect Dis. 2005:40:114-22.

11. Loeb MB, Craven S, McGeer AJ, Simor AE, Bradley SF, Low DE, et al. Risk factors for resistance to antimicrobial agents among nursing home residents. Am J Epidemiol. 2003;157:40-7.

12. Nicolle LE. The chronic indwelling catheter and urinary infection in longterm-care facility residents. Infect Control Hosp Epidemiol. 2001;22:316-21.

13. Gomes GF, Pisani JC, Macedo ED, Campos AC. The nasogastric feeding tube as a risk factor for aspiration and aspiration pneumonia. Curr Opin Clin Nutr Metab Care. 2003;6:327-33.

14. Feldman N, Adler A, Molshatzki N, Navon-Venezia S, Khabra E, Cohen D, et al. Gastrointestinal colonization by KPC-producing Klebsiella Pneumoniae following hospital discharge: duration of carriage and risk factors for persistent carriage. Clin Microbiol Infect. 2013;19:E190-6.

15. Sohn KM, Peck KR, Joo EJ, Ha YE, Kang Cl, Chung DR, et al. Duration of colonization and risk factors for prolonged carriage of vancomycin-resistant enterococci after discharge from the hospital. Int J Infect Dis. 2013;17:e240-6.

16. Scanvic A, Denic L, Gaillon S, Giry P, Andremont A, Lucet JC. Duration of colonization by methicillin-resistant Staphylococcus Aureus after hospital discharge and risk factors for prolonged carriage. Clin Infect Dis. 2001;32:1393-8.

17. Pop-Vicas A, Mitchell SL, Kandel R, Schreiber R, D'Agata EM. Multidrug-resistant gram-negative bacteria in a long-term care facility: prevalence and risk factors. J Am Geriatr Soc. 2008:56:1276-80

18. Lim CJ, Cheng AC, Kennon J, Spelman D, Hale D, Melican G, et al. Prevalence of multidrug-resistant organisms and risk factors for carriage in long-term care facilities: a nested case-control study. J Antimicrob Chemother. 2014;69:1972-80.

19. Centers for Disease Control and Prevention. Multidrug-resistant organism \& Clostridium difficile infection (MDRO/CDI) module; 2017. https:/www.cdc.gov/ nhsn/PDFs/pscManual/12pscMDRO_CDADcurrent.pdf. Accessed July 10, 2017.

20. Charlson ME, Pompei P, Ales KL, Mackenzie CR. A new method of classifying prognostic comorbidity in longitudinal studies: development and validation. J Chronic Dis. 1987:40:373-83.

21. Huang YQ, Gou R, Diao YS, Yin QH, Fan WX, Liang YP, et al. Charlson comorbidity index helps predict the risk of mortality for patients with type 2 diabetic nephropathy. J Zhejiang Univ Sci B. 2014;15:58-66.

22. Mahoney $\mathrm{Fl}$, Barthel DW. Functional evaluation: the Barthel index. Md State Med J. 1965;14:61-5.

\section{Submit your next manuscript to BioMed Central and we will help you at every step:}

- We accept pre-submission inquiries

- Our selector tool helps you to find the most relevant journal

- We provide round the clock customer support

- Convenient online submission

- Thorough peer review

- Inclusion in PubMed and all major indexing services

- Maximum visibility for your research

Submit your manuscript at www.biomedcentral.com/submit
Biomed Central 